\title{
Experimental analysis and numerical simulation of variable mass flow in horizontal wellbore
}

\author{
Jing Wen ${ }^{1}$, Ming Yang ${ }^{2,3}$, Weilin $\mathrm{Qi}^{4}$, Jing Wang ${ }^{4}$, Qian Yuan ${ }^{5}$, Wei Luo ${ }^{5 *}$ \\ ${ }^{1}$ Institute of Sedimentary Geology, Chengdu University of Technology, Chengdu Sichuan 610059, China \\ ${ }^{2}$ Research Institute of Exploration and Development, Tuha Oil Field Company, PetroChina, Hami, Xinjiang 839009 \\ ${ }^{3}$ CNPCAMERICALTD. \\ ${ }^{4}$ Research and Development Center, Tuha Oilfield Company, CNPC, Shanshan, Xinjiang, 838202, China \\ ${ }^{5}$ Laboratory of Multiphase Pipe Flow (Yangtze University),Gas Lift Innovation Center, CNPC, Wuhan 430100, China
}

Corresponding Author Email: luoruichang@163.com

https://doi.org/10.18280/ijht.360141

Received: 10 August 2017

Accepted: 12 October 2017

\section{Keywords:}

horizontal wellbore, variable mass flow, numerical simulation, experimental simulation, mixture pressure drop.

\begin{abstract}
This paper attempts to explore the variable mass flow in horizontal well from both experimental and numerical perspectives. For this purpose, a simulation model was created for single-phase liquid and gas-liquid two-phase variable mass flows in the wellbore using the FLUENT simulation software, and applied to the computational fluid dynamics (CFD) simulation of the variable mass flows in a horizontal pipe. Then, several experiments were carried out on the single phase variable mass flow in three scenarios, the unperforated horizontal pipe, the horizontal pipe with one perforation, and the horizontal pipe with two perforations. Through the analysis, the author established linear regression equations regarding the relationship of mixture pressure drops caused by the perforation-main pipe flow ratio. The main conclusions of the research are as follows: First, in both single-phase and gas-liquid two phase flows, the pressure drop across the perforation process increases with the perforationmain pipe flow ratio, under the same total flow rate. The trend is independent of the number of perforations. The simulation results agree well with the experimental data. Second, for both single-phase and gas-liquid two-phase variable mass flows, the pressure drop across the perforation process increases with the main pipe velocities, when the perforation velocities remain the same. Third, the pressure drop of the horizontal pipe with one perforation is greater than the total pressure drop of the horizontal pipe with two perforations, as long as the total single-phase fluid flow rate and the main pipe flow remain the same. The trend is exactly the opposite when the total two-phase fluid flow rate and the main pipe flow remain unchanged. Fourth, for single-phase liquid flow, the friction factor of unperforated pipe obtained by the Colebrook equation is much smaller than that calculated from the experimental data. Based on the measured data, a new friction factor of the perforated horizontal pipe was presented through data fitting. Fifth, when the total flow rate remains the same for the single-phase liquid flow, the friction factor is much higher for perforated horizontal pipe than the unperforated horizontal pipe, but the proportion of the increment is relatively small. The research findings shed valuable new light on the variable mass flow in horizontal wellbore.
\end{abstract}

\section{INTRODUCTION}

Nowadays, horizontal well development is a revolutionary technology in the oil industry. During the development, the pressure drop in the long horizontal wellbore, a determinant of well productivity, should not be ignored, especially in the horizontal wells with high formation permeability, high yield, and long horizontal interval. Neglecting the temperature change caused by heat transfer, temperature may also change in general pipe flow [1-3]. It is generally accepted that the pressure drop of wellbore variable mass flow consists of friction pressure drop, acceleration pressure drop and mixture pressure drop. However, the existing studies on pressure drop of horizontal wells have concentrated on the friction pressure drop and the acceleration pressure drop of the variable mass flow in the horizontal wellbore [4-13], failing to consider the additional impact of radial inflow on the wellbore flow. Below is a brief overview of the previous research into the variable mass flow in horizontal wells.

\subsection{Experimental analyses}

In 1992, Asheim et al. [14] performed single-phase flow simulation experiments in horizontal wellbores based on fluid dynamics. Taking water as the fluid medium, the experimental plan includes both single-hole injection and double-hole injection. The injection velocity at the edge was kept below three times the main flow rate. The experiments show that the hole injection has two main impacts on the main flow in the wellbore: the boundary layer of the wellbore wall is disturbed, resulting in variation in wall friction; the pressure energy is partially consumed to accelerate the fluid from the hole(s) to the velocity of wellbore flow. Thus, the pressure loss in horizontal wellbore was divided into the friction pressure loss and the acceleration pressure loss, and described by two specific algorithms of pressure drop.

In 1993, Su et al. [15-18] experimentally calculated the friction factor of the variable mass flow in a perforated pipe. The experiments cover both no-injection and injection 
conditions with water as the fluid medium. The horizontal wellbore pressure was split into four parts: wall friction pressure drop, acceleration pressure drop, perforation roughness pressure drop and mixed pressure drop. Then, the empirical formula of mixed pressure drop and the relative size of the other pressure drops were determined according to the experimental data.

Since 1992, Ihara et al. [19-22] have conducted a series of simulation experiments on gas-liquid two-phase flow in horizontal wellbore. With air and water as the fluid media, the wellbore flow was simulated through injection at different points in the test section. The original simulation model, only applicable to low gas-liquid ratio (injection ratio), was modified considering the effect of fluid injection on pressure drop. However, the modified model still cannot yield highly accurate results under high injection ratio.

In 1995, Plaxton et al. [23] examined the single-hole injection effect on oil-water two-phase flow using a closedloop device. According to the experiment plan, the injection fluid is water, while the main flow is oil-water two-phase flow. The injection velocities were set to more than three times the main flow rate throughout the experiment.

In 1995, Yuan et al. [24-26] simulated single-phase singlehole injection on the fluid medium of water and characterized the pressure loss induced by hole injection with the integrated resistance factor. This factor covers the wall friction, acceleration pressure drop, hole injection, and so on. Then, single-phase flow experiments with perforation injection were performed to disclose the effect of perforation density on the flow features, and the formula of integrated resistance factor was derived considering the perforation density.

In 1996, Ouyang et al. [27-28] conducted experiments on the variable mass flows in horizontal wellbore, including single-phase flow and gas-liquid two-phase flow. The flow resistance was calculated based on experimental data, and the resistance factor in radial inflow was obtained with the experimental parameters. The experimental results reveal that the resistance factor increases under the laminar flow and decreases under the turbulent flow.

In 1997, Schulkes et al. [29] carried out a variable mass single-phase flow experiment on how hole injection affects axial pressure drop. With water as the fluid medium, the pressure drop of unperforated horizontal pipe was contrasted with that of perforated horizontal pipe. The comparison shows no obvious difference between the pressure drops.

From 1998 to 2000, Zhou Shengtian [30-34] designed a simulation device for the variable mass flow in horizontal wellbore, and applied it to simulate single-hole inflow of horizontal well with the fluid medium of water. During the simulation, it is assumed that the pressure drop along the pipe includes the friction pressure drop of the pipe wall, the acceleration pressure drop and the mixing pressure drop.

From 2007 to 2011, Wang Zhiming et al. [35-38] from China University of Petroleum-Beijing conducted indoor experiments to simulate the flow in single-phase and twophase flow holes, aiming to identify the impact of perforation on the axial main flow. The single-phase fluid medium is water, the two-phase fluid medium is oil and water, and the simulation material is spiral perforated steel pipe.

In 2013, Wei Jianguang et al. [39] investigated the effect of perforation parameters and hole injection on the wall friction pressure drop, acceleration pressure drop and mixed pressure drop in horizontal wellbore through a single-phase flow experiment. The full-scale casing perforating model and the fluid medium of white oil were adopted for the experiment.

\subsection{Numerical simulations}

In 1989, Stone et al. [40] coupled reservoirs and horizontal well flow with a fully implicit, three-phase thermal recovery model. The wellbore was divided into cylindrical grid blocks, and a seepage model was constructed by Darcy's law, and mass and energy balance equations. For all phases, the wellbore model encompasses mass, momentum and energy conservation equations. The Jacobian matrix was derived through analysis and solved by a direct solver.

In 1991, Long et al. [41] predicted horizontal well productivity, and simulated the flows within and near wellbore. Considering the well block as a porous medium, different methods were presented to solve the reservoir coupled with wellbore of ultrahigh permeability. Using a mixed grid system, the simulator considers that the flow in a double porous medium obeys the Darcy's law. The simulation model can deduce pressure and saturation distributions simultaneously, but cannot explain the details of the wellbore fluid flow.

In 1991, Folefac et al. [42] described horizontal wellbore flow with a numerical model of liquid-gas flow and a 1D drift flow model. After ignoring the inertia term, the models were solved in the quasi-steady state according to the mass and momentum conservation equations of fluid flow in the wellbore and reservoir. It is concluded that the liquid supply ability of the well is overestimated without considering the pressure drop in wellbore fluid flow. This is particularly true in wells of high productivity, small radius and large perforation intervals.

In 1996, Ouyang Liangbiao et al. [43] investigated the friction, gravity and fluid inflow based on multiphase flow and fluid dynamics. The acceleration pressure drop caused by fluid inflow was also taken into account. Besides, the reservoir seepage-wellbore fluid coupling unsteady model was established and solved by iteration. Compared with popular empirical and semi-empirical methods, the model works reliably in predicting the holdup and pressure gradient of gasliquid two-phase flow.

In 2000, Zhou Haibing et al. [44] explored how single-hole injection on the flow friction resistance of perforated section, and set up the corresponding physical model based on the motion equation of the viscous fluid. Assuming that the wellbore fluid is a single-phase, steady-flow, viscous, incompressible Newtonian fluid, the scholars employed the finite-volume method to discretize the flow problem. In addition, the pressure-velocity coupling was handled by the SIMPLE algorithm, the turbulence in non-staggered grids was eliminated by momentum interpolation, and the convection term was expressed in second-order Quick format. The model of the problem was solved by the implicit method of discrete linear equations. Through the calculation, the friction factor between laminar flow and turbulent pipe flow was correlated with the Reynolds number of the cross-section and the dimensionless injection velocity of the wall.

In 2002, Zhang Qi et al. [45] theoretically analysed the flow pattern and pressure drop of gas-liquid two-phase variable mass flow in horizontal wellbore. Specifically, the Taitel and Dukler horizontal models were extended by the KelvinHelmholtz instability theory to obtain the transition discriminant of stratified flow to non-stratified flow in gasliquid two-phase flow of horizontal wellbore. On this basis, a flow pattern discrimination model was built for stratified flow, 
intermittent flow, dispersed foam flow and annular mist flow. Meanwhile, the pressure drop calculation model was derived for stratified flow and intermittent flow. The corresponding flow pattern was drawn in view of the modelling results.

In 2002, Cheng Lingsong et al. [46] established a horizontal wellbore pressure loss model based on the vertical well blackoil model and four kinds of pressure drops (i.e. friction pressure drop, acceleration pressure drop, mixed pressure drop and gravity pressure drop), and developed a novel solution called block matrix multiplication with preconditioned conjugate gradient method. The calculation results demonstrate that the proposed method has a good convergence. Thus, a software was compiled on the basis of this method.

In 2004, Wu Shuhong et al. [47] divided a horizontal wellbore into several micro-element sections without changing the total wellbore flow rate. It is assumed that the flow in a micro-element section has nothing to do with the radial flow concentrating on the interface between two adjacent sections. Thus, the flow in each section was regarded as an equal mass flow irrespective of the radial flow. On this basis, a simplified model of pipe flow was obtained in the horizontal section of equivalent seepage model. Then, the staggered-grid finite-difference method was adopted for the calculation. The mass and energy conservation equations were computed with a set of grids, and the equivalent seepage model with another set. The two sets were staggered up by half a grid, forming the staggered grids. After coupling wellbore grids with reservoir seepage grids, the wellbore flow in the horizontal section was treated as an equivalent seepage problem in porous media with some seepage capacity in reservoir numerical simulation. Finally, the parameters of horizontal wellbore were discussed by the coupling model.

In 2004, Duan Yonggang et al. [48], following the theory of unsteady seepage flow, treated the seepage and flow of horizontal wellbores as an organic whole, established a mathematical model of unsteady flow under the coupling of the reservoir and the wellbore, and derived the numerical solutions to the wellbore-reservoir coupling model. The wellbore flow model involves such complex factors as fluid friction, momentum change and mixed disturbances flowing into the wellbore wall, while the wellbore-reservoir coupling model was built by boundary integral method.

In 2005, Wang Xiaoqiu et al. [49-50] took the instability seepage model of infinite homogeneous formation as reservoir seepage model, and created the seepage pressure drop model of horizontal wellbore coupled with the formation. The horizontal wellbore pressure drop model considers the effects of wall friction pressure drop, acceleration pressure drop and mixing pressure drop. According to the coupling conditions, the horizontal wellbore and formation are in the same pressure system. Then, the pressure drop and output distribution in the horizontal wellbore were acquired by solving the coupling model in actual cases. Under the coupling of wellbore and reservoir, the results reveal the variable mass flow features of the horizontal well with perforation completion.

To sum up, there are many experimental analyses or numerical simulations on the variable mass flow in horizontal well. Nevertheless, the experimental and numerical methods are rarely combined in the same research. To make up for the gap, this paper attempts to explore the variable mass flow in horizontal well from both experimental and numerical perspectives.

\section{NUMERICAL SIMULATION ON VARIABLE MASS FLOW IN HORIZONTAL WELLBORE}

To simulate the internal flow field of the horizontal wellbore, the author established a simulation model for single-phase liquid and gas-liquid two-phase variable mass flows in the wellbore using the FLUENT simulation software.

\subsection{Flow control equation and numerical simulation method}

In a horizontal wellbore, both the single-phase liquid and gas-liquid two-phase flows satisfy the mass equation and momentum equation.

(1) Single-phase flow

The single-phase liquid in the horizontal wellbore can be viewed as an incompressible fluid, whose flow satisfies the mass and momentum equations.

The mass conservation equation can be expressed as:

$$
\frac{\partial \rho}{\partial t}+\frac{\partial\left(\rho u_{i}\right)}{\partial x_{i}}=0
$$

Where $\rho$ is the liquid density; $\mathrm{t}$ is the time; $\mu_{i}$ is the velocity in the $i$-th direction; $x_{i}$ is the spatial coordinate in the $i$-th direction.

The momentum conservation equation can be expressed as:

$$
\frac{\partial}{\partial t}\left(\rho u_{i}\right)+\frac{\partial}{\partial x_{j}}\left(\rho u_{i} u_{j}\right)=-\frac{\partial p}{\partial x_{i}}+\frac{\partial \tau_{i j}}{\partial x_{j}}+\rho g_{i}+S_{i}
$$

Where $p$ is the static pressure; $g_{i}$ is the gravity in the $i$-th direction; $\tau_{\mathrm{ij}}$ is the stress tensor; $S_{i}$ is the source of momentum. The source of momentum was considered as 0 because the formation seepage is negligible.

(2) Two-phase flow

The FLUENT simulation uses the mixture model solver: the primary phase is gas, the secondary phase is water, and the model is $\mathrm{k}-\varepsilon$ standard turbulence model. The near-wall surface was processed with a balanced wall function. The pressure and velocity were coupled by the SIMPLE algorithm. The Standard and PRESTO methods were selection for pressure coupling based on the injection velocity of the wall and the magnitude of the primary velocity. Moreover, Green-Gauss Cell-based interpolation algorithm was adopted, and the second-order regression was employed for the momentum equation and the volume fraction. In the two-phase flow model, the fluid media are water and air.

\subsection{Computational fluid dynamics (CFD) simulation}

(1) CFD model and boundary conditions

In the CFD simulation, the horizontal wellbore was simulated with a horizontal pipe. The parameters of the pipe are as follows: the inner diameter (ID) is $27.8 \mathrm{~mm}$, the length is $1 \mathrm{~m}$, and the side hole ID is $12.7 \mathrm{~mm}$. Several holes (phase angle: $180^{\circ}$ ) were opened vertically on the pipe at an interval of $30 \mathrm{~cm}$. The first top hole and the first bottom hole were $20 \mathrm{~cm}$ and $35 \mathrm{~cm}$ away from the inlet, respectively. The physical model of the horizontal wellbore is shown in Figure 1. The flow area was meshed into 1,443,610 tetrahedral grids (Figure $2)$, reaching the grid independence requirements. 


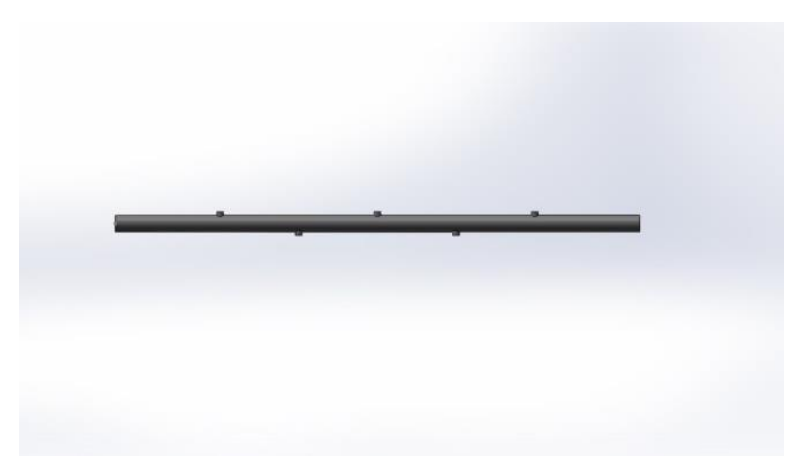

Figure 1. Physical model of horizontal wellbore

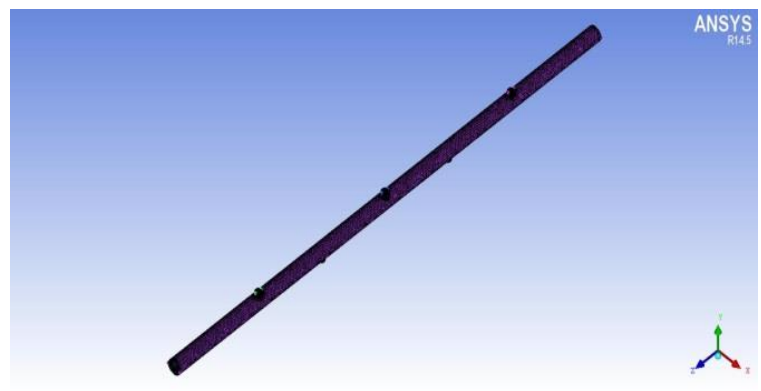

Figure 2. Meshing model of horizontal wellbore

(2) Analysis of simulation results

(1) Single-phase flow

Firstly, the single-phase flow was simulated with one hole (perforation) on the pipe. Figure 3 illustrates the variation in pressure with single perforation velocities at the total flow rate of $1.0 \mathrm{~m}^{3} / \mathrm{h}$. It is clear that the pipe pressure drop increased with the perforation velocity. The relationship between the friction factor and injection velocity ratio is presented in Figure 4.

Secondly, the single-phase flow was simulated with two perforations on the pipe. Figure 5 displays the variation in pressure with the two perforation velocities at the total flow rate of $1.0 \mathrm{~m}^{3} / \mathrm{h}$. It can be seen that the pipe pressure drop increased with the two perforation velocities under the constant total flow rate. Comparing the situation of the two perforations, it is learned that the pressure drop across the hole injection grew with the pipe flow when the two perforations have the same velocity.

Figure 6 compares the simulated pressure of the single perforation plan and that of the double perforation plan. It is observed that the single perforation plan has a greater pressure drop than the double perforation plan under fixed total flow rate and the pipe flow.

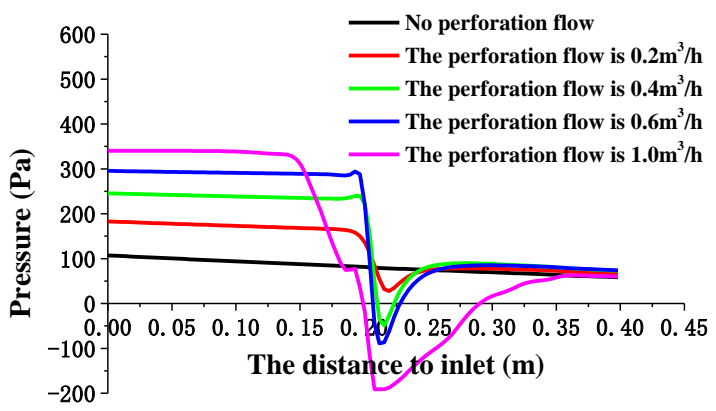

Figure 3. Pressure variation curves (Total volume: $1.0 \mathrm{~m}^{3} / \mathrm{h}$ )

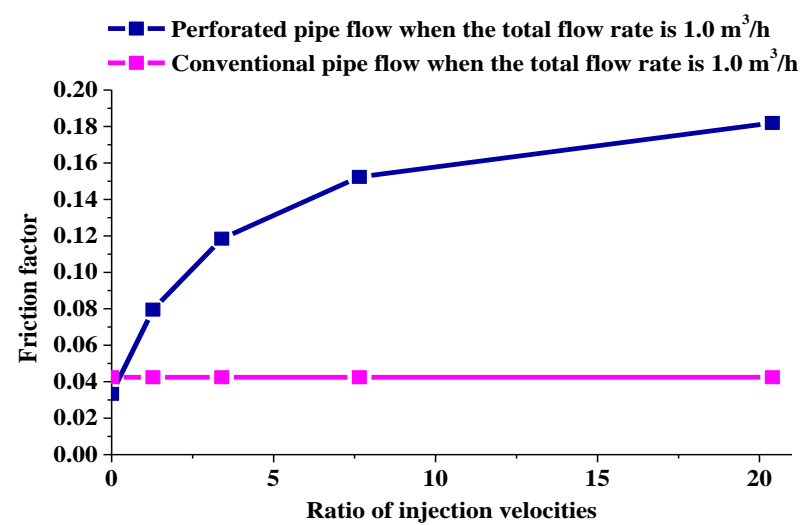

Figure 4. Relationship between the friction factor and injection velocity ratio

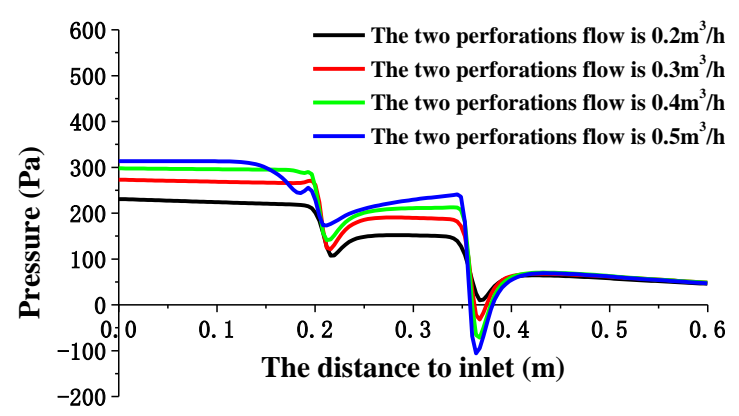

Figure 5. Pressure variation curves (Total volume $=1.0 \mathrm{~m}^{3} / \mathrm{h}$ )

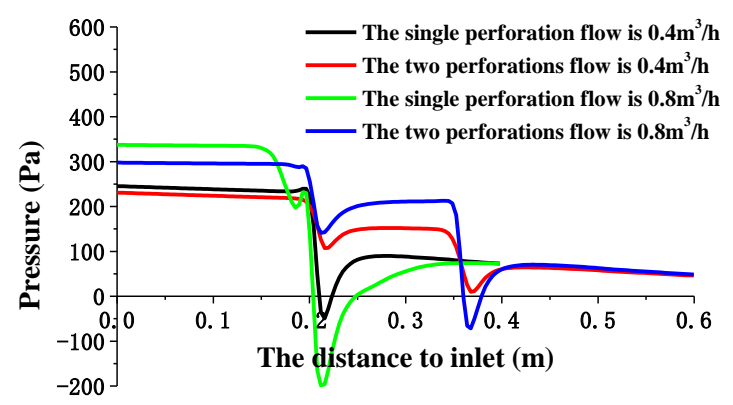

Figure 6. Pressure variation curves (Total volume $=1.0 \mathrm{~m}^{3} / \mathrm{h}$ )

(2) Gas-liquid two-phase flow

Firstly, the two-phase flow was simulated with one perforation on the pipe. Figure 7 illustrates the variation in pressure with single perforation velocities at the total flow rate of $1.0 \mathrm{~m}^{3} / \mathrm{h}$ and the gas-liquid ratio of $50 \mathrm{~m}^{3} / \mathrm{m}^{3}$.

Secondly, the two-phase flow was simulated with two perforations on the pipe. Figure 8 displays the variation in pressure with the two perforation velocities at the total flow rate of $1.0 \mathrm{~m}^{3} / \mathrm{h}$ and the gas-liquid ratio of $50 \mathrm{~m}^{3} / \mathrm{m}^{3}$.

Figure 9 compares the simulated pressure of the single perforation plan and that of the double perforation plan at the total flow rate of $1.0 \mathrm{~m}^{3} / \mathrm{h}$ and the gas-liquid ratio of $50 \mathrm{~m}^{3} / \mathrm{m}^{3}$. As shown in Figures 7-9, the pipe pressure drop increased with the perforation flow rate(s), and the single perforation plan has a smaller pressure drop than the double perforation plan under fixed total flow rate and the pipe flow. 


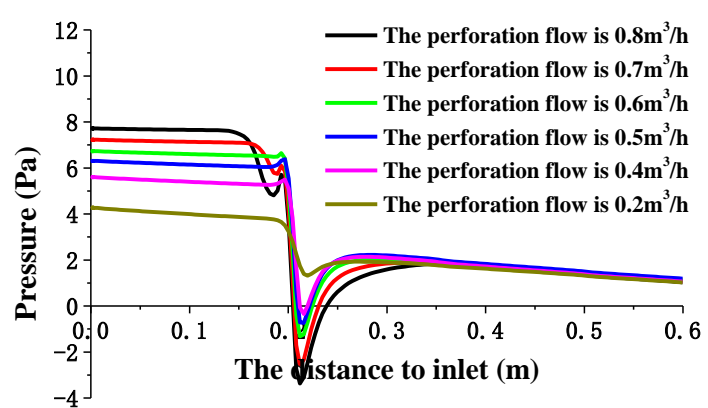

Figure 7. Pressure variation curves (Total liquid volume $=1.0 \mathrm{~m}^{3} / \mathrm{h}$ )

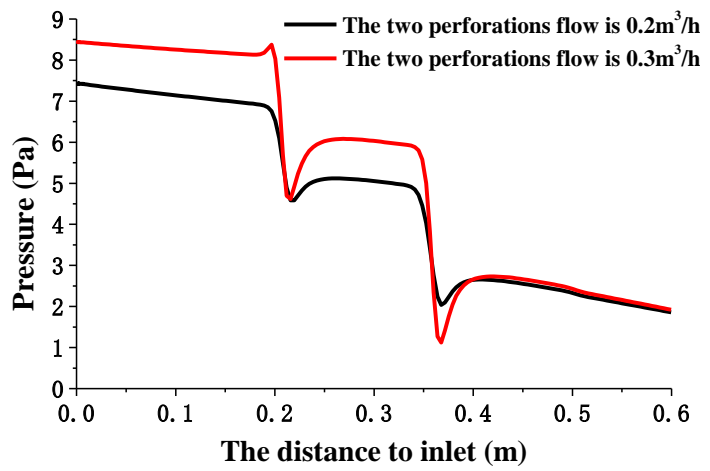

Figure 8. Pressure variation curves (Total liquid volume $=1.0 \mathrm{~m}^{3} / \mathrm{h}$ )

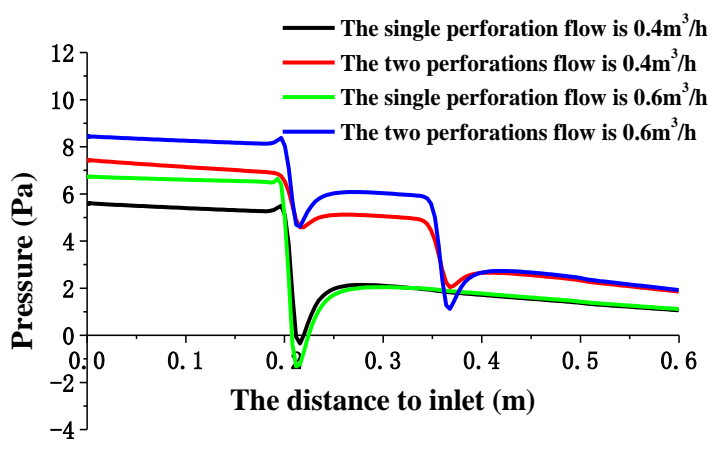

Figure 9. Pressure variation curves (Total liquid volume $=1.0 \mathrm{~m}^{3} / \mathrm{h}$ )

\section{EXPERIMENTAL ANALYSIS}

\subsection{Experimental instruments and procedure}

Except for the 10m-long test pipe section, the experimental instruments and procedure are as specified in Reference [36].

The pipe diameter of the test pipe section is DN28 and that of the side pipe is DN13. The entire test facility is about $16 \mathrm{~m}$ long. The transparent and visible side pipe was inserted into the test pipe section. The parameters of the main instruments are listed in Table 1 below.

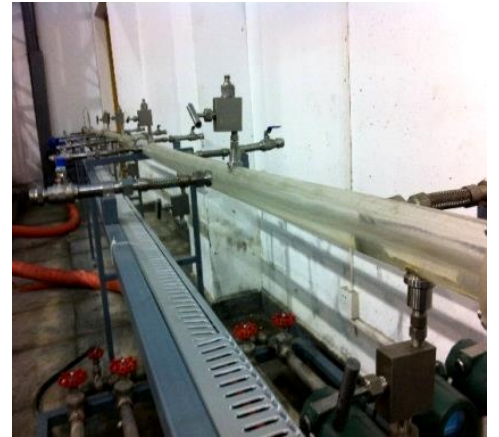

Figure 10. Experimental instruments

Table 1. Parameters of the main instruments

\begin{tabular}{ccc}
\hline Devices & $\begin{array}{c}\text { measurement } \\
\text { ranges }\end{array}$ & Accuracy \\
\hline $\begin{array}{c}\text { Turbine flowmeter of liquid } \\
\text { flow of main pipe }\end{array}$ & $0-6\left(\mathrm{~m}^{3} / \mathrm{h}\right)$ & $\pm 0.5 \%$ \\
\hline Differential pressure gauge & $0-15 \mathrm{KPa}$ & $\leq 1 \mathrm{KPa}$ \\
\hline $\begin{array}{c}\text { Thermal mass flowmeter of } \\
\text { gas flow of main pipe }\end{array}$ & $0-10\left(\mathrm{~m}^{3} / \mathrm{min}\right)$ & $\pm 1 \%$ \\
\hline $\begin{array}{c}\text { Turbine flowmeter of liquid } \\
\text { flow of side pipe }\end{array}$ & $0-1.2\left(\mathrm{~m}^{3} / \mathrm{h}\right)$ & $\pm 0.5 \%$ \\
\hline $\begin{array}{c}\text { Thermal mass flowmeter of } \\
\text { gas flow of side pipe }\end{array}$ & $0-2\left(\mathrm{~m}^{3} / \mathrm{min}\right)$ & $\pm 1 \%$ \\
\hline
\end{tabular}

\subsection{Experimental range and friction factor calculation}

(1) Experimental medium and range

Water was selected as the medium for the experiment on single-phase variable mass flow, while air and water as the media for the experiment on the gas-liquid two-phase variable mass flow. The pressure measuring section is $2 \mathrm{~m}$ long. The experimental range is shown in Table 2.

Table 2. Experimental range

\begin{tabular}{|c|c|c|c|}
\hline $\begin{array}{c}\text { Liquid phase } \\
\text { flow rate } \\
\left(\mathrm{m}^{3} / \mathbf{h}\right)\end{array}$ & $\begin{array}{c}\text { Pipe } \\
\text { diameter } \\
(\mathbf{m})\end{array}$ & $\begin{array}{c}\text { Liquid phase } \\
\text { flow velocity } \\
(\mathrm{m} / \mathrm{s})\end{array}$ & Type \\
\hline $0.45-7$ & 0.028 & 0.194-3.159 & $\begin{array}{c}\text { Single-phase } \\
\text { horizontal pipe } \\
\text { flow }\end{array}$ \\
\hline $2-7$ & 0.028 & $0.903-3.159$ & $\begin{array}{c}\text { Single-phase } \\
\text { variable mass flow }\end{array}$ \\
\hline
\end{tabular}

(2)Calculation of friction factor

(1) The friction factors were calculated as four times the value of Colebrook equation [39].

The friction factors under different Reynolds numbers were calculated based on Colebrook equation. Since the factors are $1 / 4$ of the Darcy-Weisbach friction factor, they should be multiplied by four when used in fluid dynamics.

(2) The friction factors were calculated based on the pressure drop measured in the experiment.

The energy conservation can be expressed as:

$\left(\frac{V^{2}}{2 g}+z+\frac{p}{\rho g}\right)_{1}=\left(\frac{V^{2}}{2 g}+z+\frac{p}{\rho g}\right)_{2}+h_{L}$

where $h_{L}=h_{f}+h_{m}$ is the head loss, with $h_{f}$ is the linear loss and $h_{m}$ is the local loss. For the steady flow in horizontal pipe, there is only the linear loss, that is, $h_{L}=h_{f}$.

For single-phase flow in horizontal pipe, the potential 
energy term and kinetic energy term remain unchanged. In other words, the pressure energy loss equals the linear loss. Hence, the friction factor can be expressed as:

$$
f=\frac{2 \Delta p d}{\rho v^{2} l}
$$

Where $\triangle p$ is the measured pressure drop.

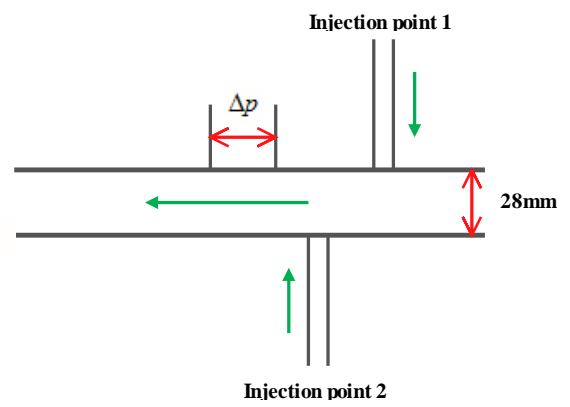

Figure 11. Experimental section of variable mass flow

The case is different with the variable mass flow in horizontal pipe. The experimental section of the variable mass flow is presented in Figure 11. The total measured pressure drop can be divided into three parts, namely, linear pressure drop, acceleration pressure drop and mixture pressure drop. The calculation formulas for linear and acceleration pressure drops are readily available. In this experiment, the linear pressure drop occurs in the downstream end, while the linear pressure drop occurs in the perforated section.

$\Delta p_{f}=\frac{1}{2} \rho f \frac{l}{D} v_{2}^{2}$

where $f$ and $v_{2}$ are fluid friction factor and velocity at the downstream end of the perforated section.

The acceleration pressure drop can be expressed as:

$$
\Delta p_{a c c}=\rho v_{1}^{2}\left[2 \frac{v_{p}}{v_{1}} \frac{A_{p}}{A_{1}}+\left(\frac{v_{p}}{v_{1}} \frac{A_{p}}{A_{1}}\right)^{2}\right]
$$

Where $v_{l}$ is the upstream velocity of the perforated section; $v_{p}$ is the perforation flow rate; $A_{l}$ is the cross-section area of test pipe section; $A_{p}$ is the cross-section area of hole and $\rho$ is fluid density.

The mixture pressure drop can be expressed as:

$$
\Delta p_{m}=\Delta p-\Delta p_{f}-\Delta p_{a c c}
$$

where $\triangle p_{m}$ is the mixture pressure drop; $\triangle p$ is the measured pressure drop.

The pressure drop section starts from the downstream end and terminates at the injection point. In the section, the fluid velocity in the downstream of the injection point is extremely small. Hence, the acceleration pressure drop along the axis can be viewed as zero, and the calculation formula for the mixture pressure drop can be expressed as:
$\Delta p_{m}=\Delta p-\Delta p_{f}$

(3) Calculation of the friction factor of horizontal pipe flow The friction factor of the horizontal pipe flow was calculated by steps (1) and (2). The results are displayed in Figure 12 .

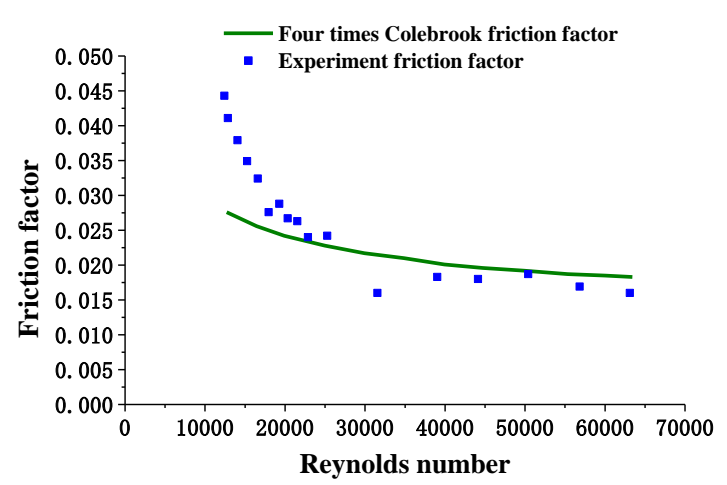

Figure 12. Comparison of experimental friction factor and Colebrook friction factor

As shown in Figure 12, the friction factor obtained by the Colebrook equation is much smaller than that derived from the measured results. The slight difference originates from the inconsistency between the perforated horizontal pipe flow in our experiment and the standard flow in unperforated horizontal pipe. Due to the side holes, the roughness of the test pipe section is changed, such that the pipe flow no longer conform to the classical Colebrook equation of horizontal pipe flow.

In order to compare the variable mass flows in perforated and unperforated horizontal pipes, the friction factors of the flows in perforated horizontal pipe were fitted under different Reynolds numbers. The fitting results were obtained as:

When $\operatorname{Re}<32,000$,

$\frac{1}{\sqrt{\lambda}}=5.305 \ln (\operatorname{Re} \sqrt{\lambda})-36.866$

When $\operatorname{Re} \geq 32,000$,

$\frac{1}{\sqrt{\lambda}}=-0.179 \ln (\operatorname{Re} \sqrt{\lambda})+9.171$

\subsection{Experimental analysis of single-phase variable mass flow}

(1)The variation in the friction factor of perforated horizontal pipe

Several single-phase variable mass flow experiments were conducted for unperforated horizontal pipe, the horizontal pipe with one perforation, and the horizontal pipe with two perforations. Furthermore, several more experiments were performed for horizontal pipe with one perforation at different injection ratios and the same total flow rates.

(1) Unperforated horizontal pipe

The friction factor calculated based on the measured pressure drop is referred to as the experimental friction factor, 
while the friction factor for unperforated horizontal pipe calculated by formulas (9) and (10) is referred to as the fitting friction factor.

According to the computing results (Figure 13), there is no side hole inflow in the test pipe section, and the fitting friction factor agrees well with the experimental friction factor.

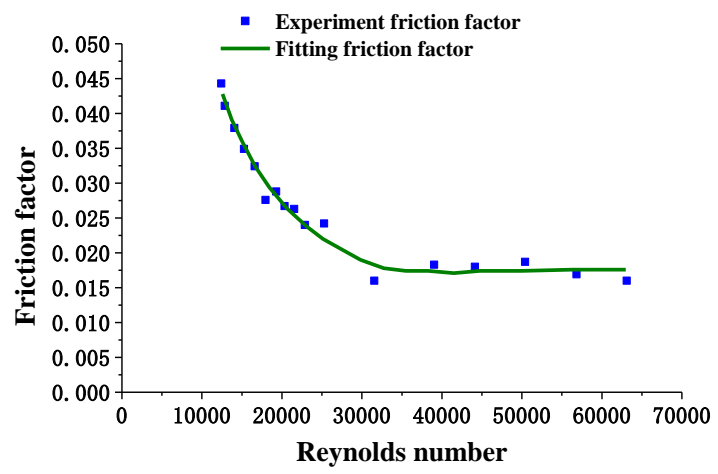

Figure 13. Experimental and fitting friction factors of unperforated horizontal pipe

\section{(2)Horizontal pipe with one perforation}

In the horizontal pipe with one perforation, there exist an axial main flow and a side hole inflow at the same time. It is assumed there is no mixture pressure drop, in light of the computing method for the friction factor of variable mass horizontal pipe. Thus, the pressure drop equals the measured pressure drop and additional friction factor of the experiment. The fitting friction factor was computed in the same way as that for the unperforated horizontal pipe. Figure 14 shows both the experimental and fitting friction factors. It is clear that the pressure drop of the horizontal pipe with one perforation is significantly higher than that of the unperforated pipe.

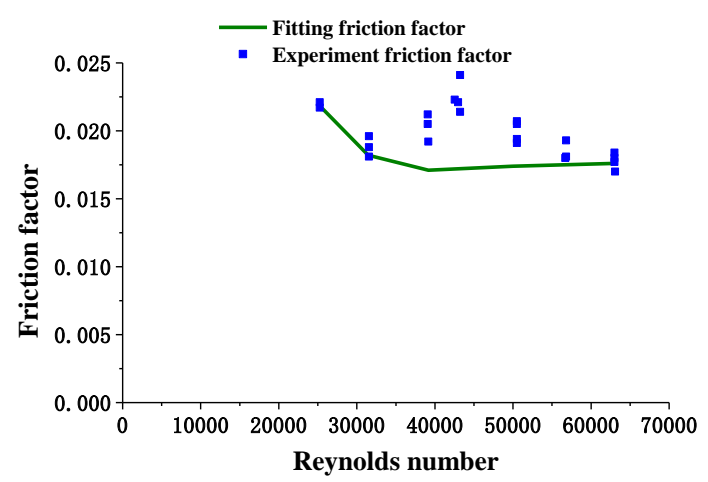

Figure 14. Experimental and fitting friction factors of horizontal pipe with one perforation

\section{(3) Horizontal pipe with two perforations}

For horizontal pipe with two perforations, the experimental and fitting friction factors were obtained in the same way as that of the horizontal pipe with one perforation. The results are presented in Figure 15.

As shown in Figures 13-15, there are significant differences between the horizontal pipe with one or two perforations and the unperforated horizontal pipe, provided that the flow rate falls in $1-5 \mathrm{~m}^{3} / \mathrm{h}$ (for $4-1 / 2$ in casing, $331.1-1,655.5 \mathrm{~m}^{3} / \mathrm{d}$ ) and the total flow rates are the same. Comparatively speaking, the contrast between the horizontal pipe with two perforations and the unperforated horizontal pipe is sharper than that between horizontal pipe with one perforation and the unperforated horizontal pipe. The friction factor is much higher for perforated horizontal pipe than the unperforated horizontal pipe, but the proportion of the increment is relatively small.

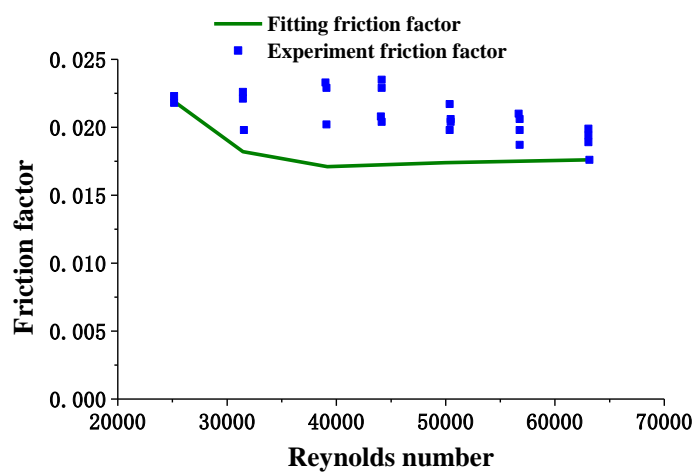

Figure 15. Experimental and fitting friction factors of horizontal pipe with two perforations

(2)Variation in friction factor with injection velocity ratios at the same total flow rate

The variation in friction factor with injection velocity ratios was examined under the same total flow rate. The results of varied total flow rates and injection velocity ratios were calculated based on the measured data for horizontal pipe with one perforation.

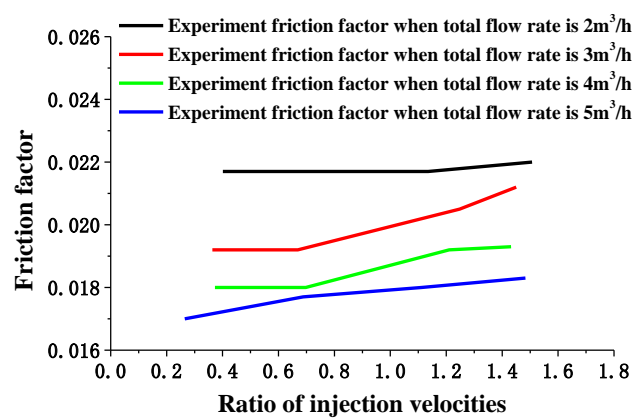

Figure 16. Experimental friction factors at various injection velocity ratios

It can be seen that the experimental friction factor generally increased with the injection velocity ratio. The trend is the same with the numerical simulation results (Figure 4). With one perforation, the friction factor acquired from the measured pressure drop decreased with the increase in the total flow rate.

(3)Additional pressure drop induced by variable mass flow

Based on the measured data of the horizontal pipe with one perforation, the mixture pressure drop was computed with the fitting friction factor and mixture pressure drop formula (11).

$$
\Delta p_{\text {mix }} / \Delta x=\frac{1}{2}\left(-2.718+3.279 v-0.943 v^{2}+1.004\left(\frac{v_{p}}{v}\right)-0.17\left(\frac{v_{p}}{v}\right)^{2}\right)
$$

Where $\triangle x$ is the length that generates $\triangle p_{\text {mix }}(\mathrm{KPa})$. The value of $\triangle x$ was set to $2 \mathrm{~m}$ in our experiments.

Then, the main pipe flow rate, the perforation flow rate, and the perforation-main pipe flow ratio were computed, and the divisor regression analysis was performed on the SPSS 
software. The linear regression equations were derived with the dependent variable of the mixture pressure drop, and the independent variables of the main pipe flow rate, the square of the main pipe flow rate, the perforation-main pipe flow ratio, and the square of the perforation-main pipe flow ratio.

\section{CONCLUSIONS}

Numerical simulations were performed on both singlephase and two-phase variable mass flows in horizontal wellbore. The following two conclusions were drawn from the simulation: First, in both single-phase and gas-liquid two phase flows, the pressure drop across the perforation process increases with the perforation-main pipe flow ratio, under the same total flow rate. The trend is independent of the number of perforations. The simulation results agree well with the experimental data. Second, for both single-phase and gasliquid two-phase variable mass flows, the pressure drop across the perforation process increases with the main pipe velocities, when the perforation velocities remain the same.

The pressure drop of the horizontal pipe with one perforation is greater than the total pressure drop of the horizontal pipe with two perforations, as long as the total single-phase fluid flow rate and the main pipe flow remain the same. The trend is exactly the opposite when the total twophase fluid flow rate and the main pipe flow remain unchanged.

For single-phase liquid flow, the friction factor of unperforated pipe obtained by the Colebrook equation is much smaller than that calculated from the experimental data. Based on the measured data, a new friction factor of the perforated horizontal pipe was presented through data fitting.

The experimental results show that, when the total flow rate remains the same for the single-phase liquid flow, the friction factor is much higher for perforated horizontal pipe than the unperforated horizontal pipe, but the proportion of the increment is relatively small.

Based on the experimental results on single-phase variable mass flow, the linear regression equation was established through data fitting for the mixture pressure drops caused by the perforation-main pipe flow ratio.

\section{ACKNOWLEDGMENT}

The authors thank Luo Wei for serving as the corresponding author for the article. The authors would like to acknowledge the support of the National Natural Science Found Project (NO. 61572084) and National Key Scientific and Technological Project (2017ZX05030-005, 2016ZX05056004-002, 2016ZX05046004-003) for key projects.

\section{REFERENCES}

[1] Medina YC, Khandy NH, Fonticiella OMC, Morales OFG. (2017). Abstract of heat transfer coefficient modelation in single-phase systems inside pipes, Mathematical Modelling of Engineering Problems 4(3): 126-131. https://doi.org/10.18280/mmep.040303

[2] Gao XQ, Zhu YH, Wang JJ, Jin YH. (2017). Effects of elbow structure of natural gas pipeline on condensation of water vapor, International Journal of Heat and
Technology

35(1):

626-632.

https://doi.org/10.18280/ijht.350320

[3] Bhattacharyya S, Das S, Sarkar A, Guin A, Mullick A. (2017). Numerical simulation of flow and heat transfer around hexagonal cylinder, International Journal of Heat and 3echnology 35(2): 360-363. https://doi.org/10.18280/ijht.350218

[4] Stone TW, Edmunds NR, Kristoff BJ. (1989). A comprehensive wellbore/reservoir simulator. The 1989 SPE Symposium on Reservoir Simulation, Houston 141153. https://doi.org/10.2118/18419-MS

[5] Dikken BJ. (1990). Pressure drop in horizontal wells and its effect on production performance. Journal of Petroleum Technology 42(11): 1426-1433. https://doi.org/10.2118/19824-PA

[6] Doan Q, Ali SMF, George AE. (1992). Scaling criteria and model experiments for horizontal wells. Journal of Canadian Petroleum Technology 31(9): 57-65. https://doi.org/10.2118/92-09-07

[7] Novy RA. (1995). Pressure drops in horizontal wells: when can they be ignored? SPE Reservoir Engineering 10(10): 29-35. https://doi.org/10.2118/24941-PA

[8] Landman MJ, Goldthorpe WH. (1991). Optimization of perforation distribution for horizontal wells. SPE AsiaPacific Conference, November 567-576. https://doi.org/10.2118/23005-MS

[9] Landman MJ. (1994). Analytic modelling of selectively perforated horizontal wells. Journal of Petroleum Science \& Engineering 10(3): 179-188. https://doi.org/10.1016/0920-4105(94)90079-5

[10] Marett BP, Landman MJ. (1993). Optimal perforation design for horizontal wells in reservoirs with boundaries. The SPE Asia Pacific Oil and Gas Conference and Exhibition Singapore 397-406. https://doi.org/10.2118/25366-MS

[11] Ozkan E, Sarica C, Haciislamoglu M, Raghavan R. (1995). Effect of conductivity on horizontal well pressure behavior. SPE Advanced Technology 3(1): 85-94. https://doi.org/10.2118/24683-PA

[12] Sarica C, Haciislamoglu M, Raghavan R, Brill JP. (1994). Influence of wellbore hydraulics on pressure behavior and productivity of horizontal gas wells. The SPE 69th Annual Technical Conference and Exhibition, USA: New Orleans LA 237-247. https://doi.org/10.2118/28486-MS

[13] Suzuki K. (1997). Influence of wellbore hydraulics on horizontal well pressure transient behavior. Spe Formation Evaluation 12(3): 175-181. https://doi.org/10.2118/24684-PA

[14] Asheim H, Kolnes J, Oudeman P. (1992). A flow resistance correlation for completed wellbore. Journal of Petroleum Science \& Engineering 8(2): 97-104. https://doi.org/10.1016/0920-4105(92)90048-6

[15] Su Z, Gudmundsson JS. (1993). Friction Factor of Perforation Roughness in Pipes. SPE Annual Technical Conference and Exhibition, Houston, Texas 100-108. https://doi.org/10.2118/26521-MS

[16] Su Z, Gudmundsson JS. (1994). Pressure drop in perforated pipes: experiments and analysis. SPE Asia Pacific Oil and Gas Conference, Melbourne, Australia 55-58. https://doi.org/10.2118/28800-MS

[17] $\mathrm{Su} \mathrm{Z.} \mathrm{(1996).} \mathrm{Pressure} \mathrm{drop} \mathrm{in} \mathrm{perforated} \mathrm{pipes} \mathrm{for}$ horizontal wells. Ph.D. dissertation. Norwegian University of Science and Technology, rondheim, 
Norway.

[18] Su Z, Gudmundsson JS. (1998). Perforation inflow reduces frictional pressure loss in horizontal wellbores. Journal of Petroleum Science \& Engineering 19(3-4): 223-232. https://doi.org/10.1016/S0920-4105(97)000478

[19] Ihara M, Yanni K, Yakao S. (1995). Two-phase flow in horizontal wells. SPE Production\&Facilities 11: 249255. https://doi.org/10.2118/24493-PA

[20] Ihara M, Brill JP, Shoham O. (1992). Experimental and theoretical investigation of two-phase flow in horizontal wells. SPE Annual Technical Conference and Exhibition, Washington, DC, USA 57-67. https://doi.org/10.2118/24766-MS

[21] Ihara M, Shimizu N. (1993). Effect of accelerational pressure drop in a horizontal wellbore. The 68th Annual Technical Conference and Exhibition, Houston, Texas 125-138. https://doi.org/10.2118/26519-MS

[22] Ihara M, Kikuyama K, Mizuguchi K. (1994). Flow in horizontal wellbores with influx through porous walls. SPE Technical Conference and Exhibition, New Orleans, Louisiana 57-67. https://doi.org/10.2118/28485-MS

[23] Plaxton B, Ali SF. (1995). Pipeflow experiments for the analysis of pressure drop in horizontal wells. Annual Technical Meeting, Calgary, Alberta 635-650. https://doi.org/10.2118/96-64

[24] Yuan H, Sarica C, Miska S, Brill JP. (1997). An experimental and analytical study of single-phase liquid flow in a horizontal well. Journal of Energy Resources Technology 119(1): 20-25 https://doi.org/10.1115/1.2794217

[25] Yuan H, Sarica C, Brill JP. (1996). Effect of perforation density on single phase liquid flow behavior in horizontal wells. Spe Production \& Facilities 14(3): 603-612. https://doi.org/10.2118/57395-PA

[26] Yuan H, Sarica C, Brill JP. (1998). Effect of completion geometry and phasing on single-phase liquid flow behavior in horizontal wells. SPE Annual Technical Conference and Exhibition, New Orleans, Louisiana 93104. https://doi.org/10.2118/48937-MS

[27] Ouyang LB, Aziz K. (2000). A homogeneous model for gas-liquid flow in horizontal wells. Journal of Petroleum Science \& Engineering 27(3): 119-128. https://doi.org/10.1016/S0920-4105(00)00053-X

[28] Ouyang LB, Petalas N, Arbabi S, Schroeder DE, Aziz K. (1998). An experimental study of single-phase and twophase fluid flow in horizontal wells.SPE Western Regional Meeting, Bakersfield, USA. https://doi.org/10.2118/46221-MS

[29] Schulkes R, Utvik OH, Rindc T. (1997). Pressure drop in horizontal wells: multi-phase flow with radial inflow. 8th International Conference Multiphase 97, Cannes, France 45-55.

[30] Zhou ST, Zhang Q, Li MZ, Wang WY. (1998). Experimental study on variable mass fluid flow in horizontal wells. Journal of the University of Petroleum, China 22(5): 53-55.

[31] Zhou S, Zhang Q, Li M. (2002). The advances on the variable mass flow in horizontal wells. Advances in Mechanics 32(1): 119-127.

[32] Zhou ST, Zhang Q, Li MZ, Wang WY. (2000). An experimental study for horizontal well flow under the influence of the inflow through perforation. Journal of Experimental Mechanics 15(3): 306-310.
[33] Shengtian BZ, Zhang Q, Li M, Xue J. (1998). Experimental study on resistance factors of perforated horizontal pipes. Oil Drilling \& Production Technology 20(5): 52-55.

[34] Zhou ST, Zhang Q. (1998). A model for computing twophase flow pressure in horizontal wellbore. Journal of the University of Petroleum, China 22(4): 43-44.

[35] Wang ZM, Zhao TF, Xu L. (2003). Pressure drop of variable mass flow in horizontal perforated well bore. Shiyou Daxue Xuebao 27(1): 41-44.

[36] Wang ZM, Xiao JN, Wang XQ, Wei JG. (2011). Experimental study for pressure drop of variable mass flow in horizontal well. Journal of Experiments in Fluid Mechanics 25(5): 26-29.

[37] Wang ZM, Zhang SJ, Xue L, Wang XQ. (2007). Pressure drop of variable mass flow in perforation completion of horizontal wellbore. Oil Drilling \& Production Technology 29(3): 4-7.

[38] Wang ZM, Xiao JN, Wei JG, Wang XQ, Shi WJ. (2011). Study on pressure drop of oil-water vary mass dispersed flow in horizontal wellbore. Chinese Journal of Hydrodynamics 26(3): 284-288.

[39] Wei J, Wang X, Chen H, Zhang Q. (2013). Experiment of complex flow in full size horizontal wells with perforated completion. Petroleum Exploration \& Development 40(2): 220-225.

[40] Stone TW, Edmunds NR, Kristoff BJ. (1989). A comprehensive wellbore/reservoir simulator. SPE Symposium on Reservoir Simulation, Houston, Texas 141-153. https://doi.org/10.2118/18419-MS

[41] Long N, Collins D, Sharma R. (1991). Seventh spe comparative solution project: modelling of horizontal wells in reservoir simulation. 11th SPE Symposium on Reservoir Simulation, Anaheim, California U.S.A 195218. https://doi.org/10.2118/21221-MS

[42] Folefac AN, Archer JS, Issa RI, Arshad AM. (1991). Effect of pressure drop along horizontal wellbores on well performance. Offshore Europe, Aberdeen, United Kingdom 549-560. https://doi.org/10.2118/23094-MS

[43] Ouyang LB, Arbabi S, Aziz K. (1996). General wellbore flow model for horizontal, vertical, and slanted well completions. Spe Journal 3(2): 124-133. https://doi.org/10.2118/36608-PA

[44] Haibing BZ, Cui G, Huang X. (2000). Numeric simulation study of flow loss for variable mass flow in horizontal well: part oneflow in single hole. Oil Drilling \& Production Technology 22(2): 42-45.

[45] Zhang Q, Zhou ST, Wu N, Li MZ. (2002). Laws of gasliquid two-phase variable mass flow in horizontal wellbore. Journal of the University of Petroleum China 26(6): 46-49.

[46] Cheng LS, Lan JC. (2002). The method of reservoir numerical simulation when considering the pressuredrop alone the horizontal well-bore. Acta Petrolei Sinica 23(1): 67-71.

[47] Wu SH, Liu XE, Guo SP. (1999). A simplified model of flow in horizontal wellbore. Petroleum Exploration and Development 26(4): 64-65.

[48] Chen W, Duan YG, Huang C, Zhang Y. (2004). Transient performance prediction of horizontal well with coupled in wellbore and reservoir part 1: mathematic model. Journal of Southwest Petroleum Institute 26(1): 23-25.

[49] Wang XQ, Wang ZM, Wei JQ. (2005). Investigation of 
variable mass flow in horizontal well with perforation completion coupling reservoir. Journal of hydrodynamics 20(3): 326-331.

[50] Wang XQ, Xu J, Wang ZM. (2005). Progress in the research on variable mass flowing law in horizontal wellbore. Natural gas industry 25(4): 92-94.

\section{NOMENCLATURE}

$\begin{array}{ll}t & \text { the time, } \mathrm{s} \\ x & \text { the spatial coordinate, } \mathrm{m} \\ p & \text { the static pressure, } \mathrm{Pa} \\ g & \text { the gravity, } \mathrm{kg} \cdot \mathrm{m} . \mathrm{s}^{-2} \\ S & \text { the source of momentum, } \mathrm{Pa} / \mathrm{m} \\ V & \text { the velocity, } \mathrm{m} \cdot \mathrm{s}^{-1} \\ v & \text { the velocity, } \mathrm{m} \cdot \mathrm{s}^{-1} \\ z & \text { the height, } \mathrm{m} \\ h & \text { the head loss, } \mathrm{m} \\ \Delta p & \text { the differential pressure, } \mathrm{Pa} \\ d & \text { the diameter of pipe, } \mathrm{m} \\ D & \text { the diameter of pipe, } \mathrm{m}\end{array}$

$l \quad$ the length of pipe, $\mathrm{m}$

A the cross-section area of test pipe section, $\mathrm{m}^{2}$

Re Reynolds number

$\Delta x \quad$ the differential length, $\mathrm{m}$

\section{Greek symbols}

$\begin{array}{ll}\rho & \text { the liquid density, } \mathrm{kg} \cdot \mathrm{m}^{-3} \\ u & \text { the velocity, } \mathrm{m} \cdot \mathrm{s}^{-1} \\ \tau & \text { the stress tensor, } \mathrm{Pa} \\ \lambda & \text { the friction factor }\end{array}$

\section{Subscripts}

$\begin{array}{ll}i & \text { in the } i \text {-th direction } \\ \mathrm{f} & \text { friction factor } \\ L & \text { the loss } \\ 1,2 & \text { the upstream and downstream } \\ \mathrm{acc} & \text { acceleration } \\ \mathrm{p} & \text { perforated hole } \\ \mathrm{m}, \mathrm{mix} & \text { mixture }\end{array}$

